\title{
Laser Nanopatterning of Colored Ink Thin Films for Photonic Devices
}

\author{
Bader AlQattan, ${ }^{\dagger}$ David Benton, ${ }^{*}$ Ali K. Yetisen, ${ }^{\S \odot}$ and Haider Butt ${ }^{* \dagger}$ \\ ${ }^{\dagger}$ Nanotechnology Laboratory, School of Engineering, University of Birmingham, Birmingham B15 2TT, U.K. \\ ${ }^{\ddagger}$ Aston Institute of Photonics Technologies, Aston University, Birmingham, B4 7ET, U.K. \\ ${ }^{\S}$ Harvard-MIT Division of Health Sciences and Technology, Harvard University and Massachusetts Institute of Technology, \\ Cambridge, Massachusetts 02139, United States
}

\section{Supporting Information}
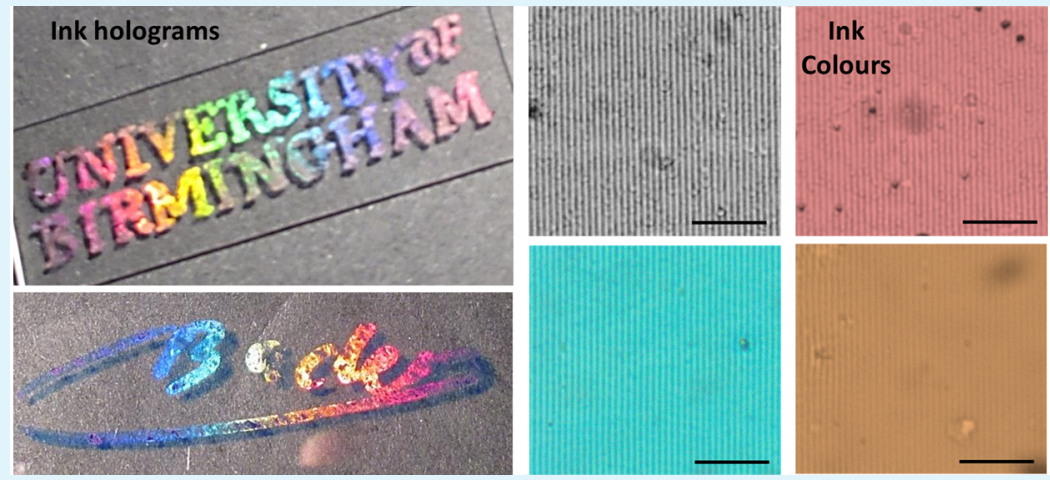

ABSTRACT: Nanofabrication through conventional methods such as electron beam writing and photolithography is timeconsuming, high cost, complex, and limited in terms of the materials which can be processed. Here, we present the development of a nanosecond Nd:YAG laser $(532 \mathrm{~nm}, 220 \mathrm{~mJ})$ in holographic Denisyuk reflection mode method for creating ablative nanopatterns from thin films of four ink colors (black, red, blue, and brown). We establish the use of ink as a recording medium in different colors and absorption ranges to rapidly produce optical nanostructures in $1 \mathrm{D}$ geometries. The gratings produced with four different types of ink had the same periodicity $(840 \mathrm{~nm})$; however, they produce distant wavelength dependent diffraction responses to monochromatic and broadband light. The nanostructures of gratings consisting of blue and red inks displayed high diffraction efficiency of certain wavelengths while the black and brown ink based gratings diffracted broadband light. These gratings have high potential to be used as low-cost photonic structures in wavelength-dependent optical filters. We anticipate that the rapid production of gratings based on different ink formulations can enable optics applications such as holographic displays in data storage, light trapping, security systems, and sensors.

KEYWORDS: Holography, laser ablation, diffraction gratings, printing, photonic devices

\section{INTRODUCTION}

Nanofabrication techniques such as electron beam lithography (EBL) or Focused Ion Beam (FIB) milling can produce meticulous nanostructures with $2-5 \mathrm{~nm}$ resolution. ${ }^{1}$ However, they require high-power energy supplies, have long producing times, and involve complex setups and equipment. ${ }^{2-5}$ In addition, they are not amenable for a variety of materials which can be directly processed. ${ }^{6}$ Coloration of different nanomaterials as metasurfaces is highly desirable to produce multifunctional and miniaturized optoelectronic devices. These devices may comprise metallic nanostructures with high polarizationindependent reflection, strong contrast, fast response time, and long-term stability. In addition they may have controllable optical absorption of a nanostructured polymer film to diffract light. ${ }^{7}$ However, these devices require layers with specific arrangement as short-range ordered nanoholes in a gold film, which affect the total cost and time, and these devices can be only used in reflection mode. ${ }^{8,9}$ Nanoparticle (NP) assemblies can also be used to create functional optical/electrical devices for sensing, electronics, displays, and catalysis. Sophisticated design patterns, high intensity and wide angle-independent structural colors are the key features of the NP-based optical devices. Ejecting ink from the nozzle, wetting, sputtering, spreading, and retracing processes can affect the precisions of the final structure of the printed nanostructures. ${ }^{10,11}$ The localized surface plasmon resonance of silver nanoplate $(\mathrm{SNP})$ substrates can be tuned from 500 to $800 \mathrm{~nm}$ in the visible spectrum. These devices have selective optical absorption and scattering behavior based of specific nanoparticle sizes. However, they required complex chemical syntheses processes. Moreover, the colors of silver films lead to strong electromagnetic scattering. ${ }^{12}$ Cubic liquid-crystal structures have been developed for electronic and photonic

Received: October 16, 2017

Accepted: October 19, 2017

Published: October 31, 2017 
(a)

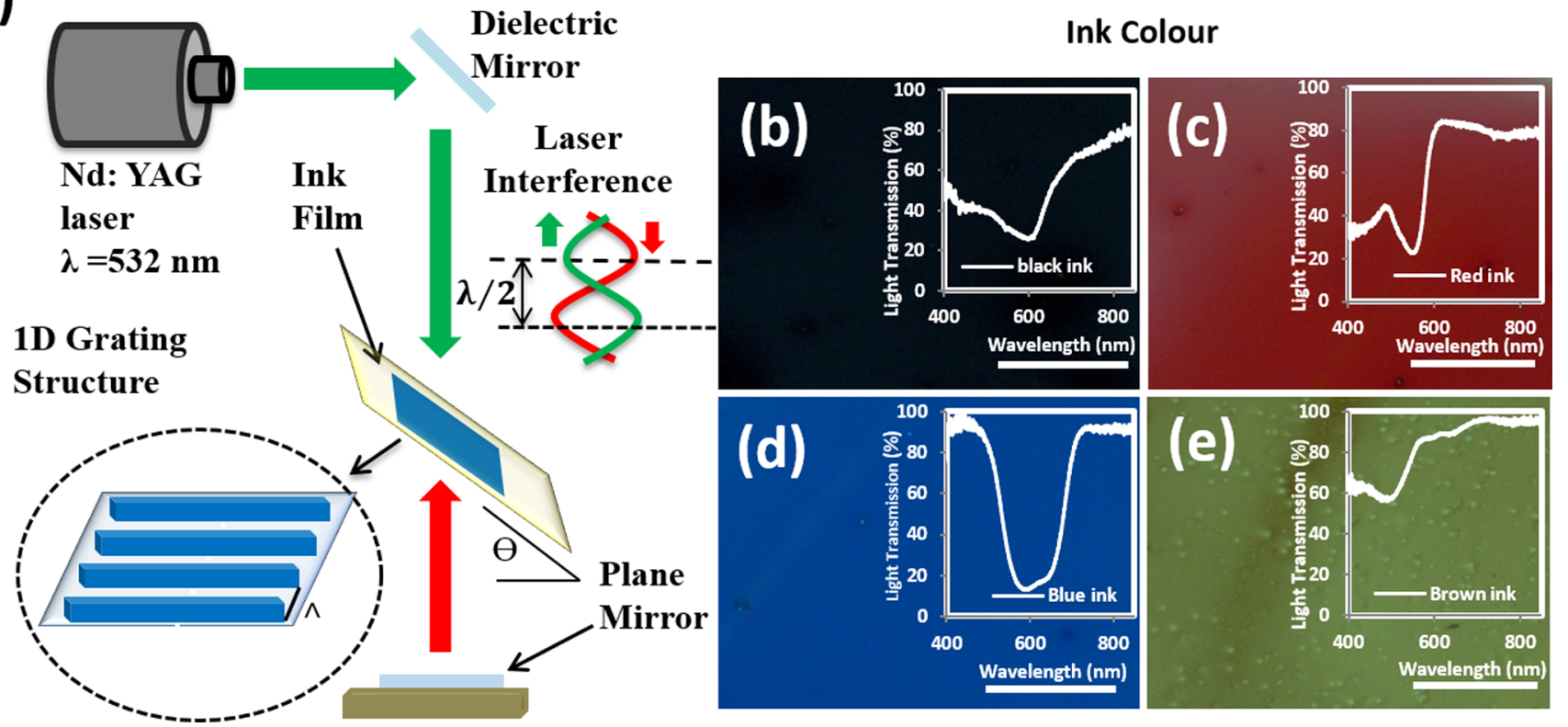

Figure 1. Fabrication of 1D patterns through nanosecond DLIP in holographic Denisyuk reflection mode. (a) Nd:YAG laser beam ( $532 \mathrm{~nm}, 3.5 \mathrm{~ns})$ was directed to a dielectric mirror and passed through a dye-based ink medium and reflected back from a plane mirror to ablate a localized region of the recording medium. Four dye-based ink mediums are (b) black, (c) red, (d) blue, and (e) brown. The light transmission measurement for each medium added on each color. Scale bar $=200 \mu \mathrm{m}$.

applications. ${ }^{13}$ Many advanced reflective photonic applications could be achieved based on the patterned crystallographic orientation of the cubic soft lattice of a blue phase liquid crystal (BPLC). The BPLC could be produced with UV irradiation and photomask which are complex and high cost. ${ }^{14}$ In addition, photolithography has been widely used to make various nanostructures for optical devices operating in the visible region ${ }^{15,16}$ However, using pulsed laser ablation requires low energy and rapid production. ${ }^{17,18}$ Recently, direct laser interference patterning (DLIP) has been used to produce nanostructures in thin materials. Such nanostructures can be patterned into $1 / 2 / 3 \mathrm{D}$ geometries, ${ }^{19-22}$ and complex patterns such as Fresnel lenses and filters. ${ }^{6,19,21}$ The expansion of this platform to a wide range of low-cost materials is highly desirable for large-scale production.

Here, we show a holographic ablation based on direct laser interference patterning method to rapidly create low-cost optical nanostructures. A Nd:YAG laser $(532 \mathrm{~nm})$ was used in holographic Denisyuk reflection mode of to create ablative interference fringes. ${ }^{19,20}$ We utilized different synthetic dyebased inks, which have different optical properties, to produce 1D photonic nanostructures. Tested ink media consisted of four ink colors (black, red, blue, and brown). Holographic DLIP allowed forming constructive antinode fringes of electromagnetic field from the standing wave to create gratings in dye-based inks. Using thick film of material (ink) causes multiple laser wave interference fringes to form the gratings. ${ }^{23} \mathrm{~A}$ thick amplitude grating invariably describes an absorption grating. Usually a thick holographic grating can be expressed as a sinusoidal absorption grating and it has different characteristics of diffraction compared to conventional gratings with sharp edges. This is because the shape and the thickness of the recording medium influences the transmission profile. ${ }^{24,25}$ Such dye-based ink gratings can be used in a myriad of devices to produce light deflection, guidance, or coupling. In the present work, the nanostructures based on the four dye-based ink gratings have been spectrally analyzed and angle-resolved measurements were performed to characterize their optical properties. This is the first demonstration of laser based nanopatterning of multiple ink formulations and their usage for wavelength dependent optical devices. The work demonstrates the possibility of nanopatterning organic and florescent materials which cannot be processed through methods such as photolithography and electron beam lithography.

\section{MATERIALS AND METHODS}

Preparation of the Ink Recording Media. Black, red, blue and brown ink colors (Staedtler Lumocolor) have refractive indexes of 1.6, $1.65,1.59$, and 1.62 respectively. Diluted ink solutions $(1: 8, \mathrm{v} / \mathrm{v}$ in ethanol) were spin coated on a $1 \mathrm{~mm}$ thick glass slides at $200 \mathrm{rpm}$ for 35 s. The inks used were permanent and had a long-term durability.

Fabrication of the Diffraction Gratings in ink. Holographic direct laser interference patterning was used in Denisyuk reflection mode. Laser beams $(\lambda=532 \mathrm{~nm})$ initially directed by a mirror traveled to an ink-based recording medium and reflected from a plane mirror to ablate the localized regions of the medium. The exposure angle of all ink films was set to $18^{\circ}$ from the surface plane of the plane mirror (object).

Spectroscopic Measurements of the Ink Gratings. The diffraction of light from 1D gratings of the four inks (black, red, blue and brown) were analyzed by normally illuminating the periodic samples with violet $(\lambda=405 \mathrm{~nm})$, green $(\lambda=532 \mathrm{~nm})$, and red $(\lambda=635$ $\mathrm{nm})$ laser beams and observing the transmitted light perpendicularly on a flat screen, placed $17 \mathrm{~cm}$ away.

Angle-Resolved Measurements of the Gratings. halogen light source (HL-2000, Ocean Optics) and a goniometer setup were used to carry out angle-resolved measurements of the diffraction efficiency of the ink gratings. Diffraction spots from the ink nanostructures were analyzed in transmission mode. Each sample was placed $17 \mathrm{~cm}$ away from the optical probe to analyze the diffraction wavelengths. A motorized rotating stage was used for broadband spectroscopic analysis of the rainbow diffraction through the nanostructure gratings. The rotation stage had $0.5^{\circ}$ steps from ranging from $-90^{\circ}$ to $+90^{\circ}$. 


\section{RESULTS AND DISCUSSION}

Holographic Fabrication of 1D Nanostructures. Figure 1a shows the diagram of the hologram recording setup in Denisyuk ablation mode. The laser beams initially directed by a mirror traveled to an ink-based recording medium and reflected from a plane mirror to ablate the localized regions of the medium. To form the gratings, four dye media were used: black ink (a combination of blue, red, and yellow dyes) (Figure $1 \mathrm{~b}$ ), red ink (Figure 1c), blue ink (Figure 1d), and brown ink (a combination of green, yellow, and red dyes) (Figure 1e).

A halogen light source was used to analyze the transmission of each ink-based recording medium (insets in Figure 1). The transmitted halogen light generated the visible range wavelengths from 400 to $850 \mathrm{~nm}$. For thick materials, the recording media could be described in terms of light absorption percentage (Absorption \% = 1-Transmission \%). The black ink had the most absorption of light in the visible range, absorbing more than $75 \%$ of all wavelengths (Figure 1b). The red ink had low absorption $(20-45 \%)$ of light in the visible range and it allowed $80 \%$ light transmission over $620 \mathrm{~nm}$ wavelengths (Figure 1c). On the other hand, the blue ink has shown high light transmission below 500 $\mathrm{nm}$ and above $700 \mathrm{~nm}$ (Figure 1d). Brown ink consisted of different dye compositions, showing high light transmission at all wavelengths. As a result, red and blue inks had sharp absorption dips in the visible spectrum; however, the black and brown inks were highly absorbent in the visible spectrum since they were composed of many colors.

Holographic DLIP was performed to produce a well-ordered grating in each ink medium, where the size of the grating pattern ranged from $0.2-1.0 \mathrm{~cm}$ in diameter (roughly the size of laser beam). The four ink media received the same laser beam energy $(210 \mathrm{~mJ})$ and they produced the same $1 \mathrm{D}$ nanostructures. The red ink had low peak absorption at $532 \mathrm{~nm}$ so it produced the smallest spot size $(0.25 \mathrm{~cm})$. The black ink had the largest laser spot size as compared to the other inks due to its high light absorption and ablation within the green region of the spectrum (Figure 2a). The laser ablation process was the result of the laser interference of two beams, which can be described by

$$
\begin{aligned}
& E_{I}=E_{01} \sin \left(k x+\omega t+\varepsilon_{I}\right) \\
& E_{R}=E_{0 R} \sin \left(k x+\omega t+\varepsilon_{R}\right) \\
& E=E_{I}+E_{R}
\end{aligned}
$$

where $E_{\mathrm{I}}$ is the incident laser wave and $\mathrm{E}_{\mathrm{R}}$ is the reflected laser wave from the plane mirror, $k$ is the propagation number or the magnitude of grating vector and $k=2 \pi / \Lambda$, and $\mathrm{x}, \omega, \mathrm{t}, \Lambda, \lambda$ represent axis plane, angular velocity, time, grating spacing, and wavelength, respectively. The initial phase or angle is sampled as $\varepsilon$. The other countenance of symbols shows the resultant wave $\mathrm{E}$ of initial and reflection waves: ${ }^{26}$

$$
\begin{aligned}
& I=I_{o} \cos \left(\frac{2 \pi \Lambda}{\lambda}\right) \\
& \Lambda=\frac{\lambda}{2 \sin (\theta)}
\end{aligned}
$$

The grating structure was created as a result of the intensity $(I)$ of laser interference, where $\left(I_{\mathrm{o}}\right)$ is the maximum laser interference intensity (eq 4). The exposure angle of all ink films was $18^{\circ}$ from the surface plane of the plane mirror (object) so the four inks had the same grating spacing of $840 \mathrm{~nm}$. The difference between the experimental results of grating spacing
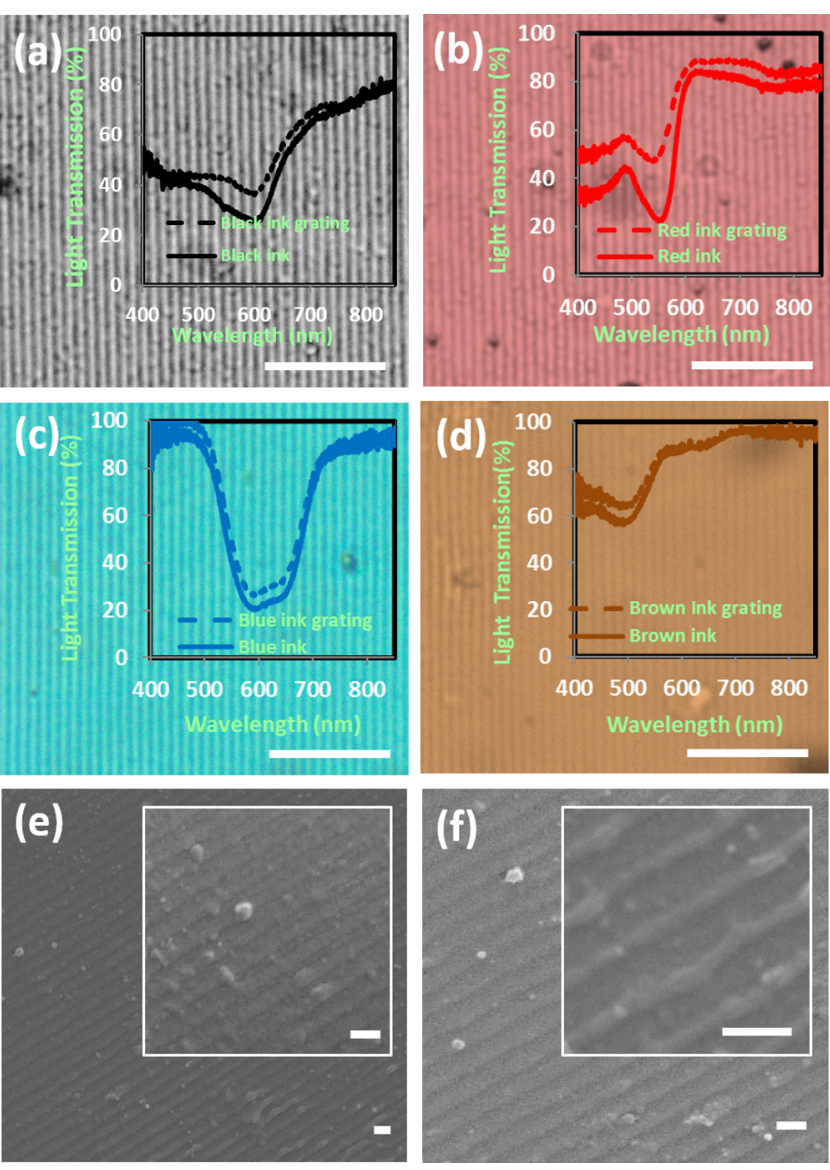

Figure 2. Optical microscopy images of 1D surface grating nanostructures fabricated by holographic DLIP. Gratings having periodicities of $840 \mathrm{~nm}$ in (a) black, (b) red, (c) blue, and (d) brown inks. SEM grating image of (e) black, (f) blue gratings. The light transmission graphs of each ink were integrated for normalization. Scale bar $=10 \mu \mathrm{m}$. SEM scale bar $=1 \mu \mathrm{m}$.

and the analytical solution was $2 \%$ (eq 5 ). The period of grating spacing can be controlled by the exposure angle $(\theta)$ between the laser beam and the tilt angle from the surface plane of the mirror (eq 5$)^{20}$

The formation of gratings on the four colored inks has increased the broadband light transmission. The average transmission ranges between 3 and $20 \%$ (Figure $2 a-d$ ). The highest percentage of transmission change was in the red ink while the lowest one was in the brown ink, whereas the transmission increased by $8 \%$ and $10 \%$ for blue and black inks, respectively (Figure 2). In general, transmission of light changed in all the ink media operating between 400 and $650 \mathrm{~nm}$ except for red ink which remained in the entire visible spectrum. The highest absorption zone of the red ink was at $559 \mathrm{~nm}$, close to the laser beam wavelength of $532 \mathrm{~nm}$. SEM images show magnifed images for the nanostructures of black and blue gratings (Figure $2 \mathrm{e}, \mathrm{f})$.

Optical Characterization of the Gratings. The created nanostructures were amplitude gratings. However, different absorption regions of the ink produced different diffraction responses. Although the standing wave field had the same source wavelength, different dye inks formed different diffraction patterns. The black grating had the same spacing as the other inks but it has different diffraction responses when illuminated with different laser beams (Figure 3). The black ink based grating 

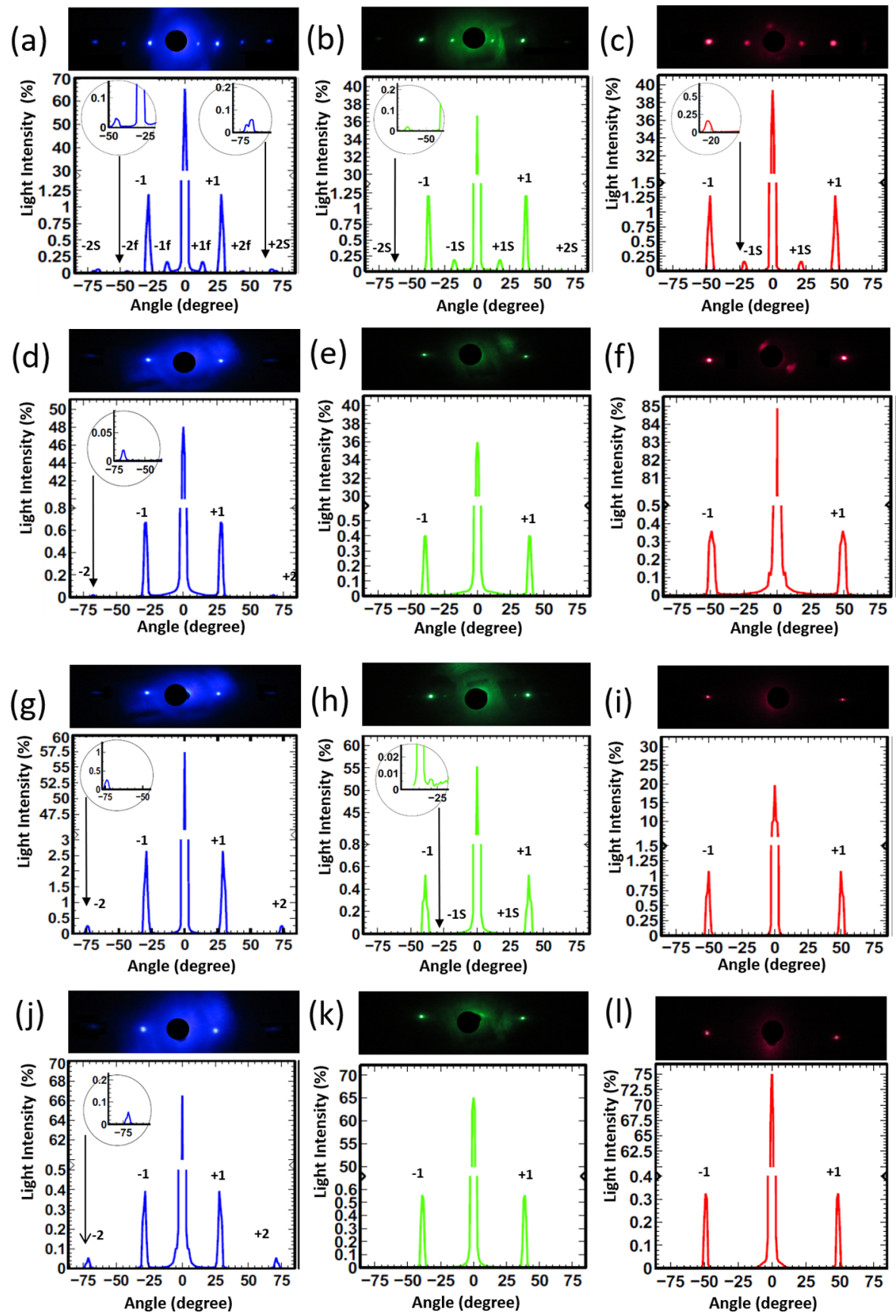

Figure 3. Diffraction from nanostructures upon illumination from three monochromatic light sources (405 nm, $532 \mathrm{and} 635 \mathrm{~nm})$ on patterned ink gratings $(\Lambda=0.84 \mathrm{~nm})(\mathrm{a}-\mathrm{c})$ Black, $(\mathrm{d}-\mathrm{f})$ red, $(\mathrm{g}-\mathrm{i})$ blue, and $(\mathrm{j}-\mathrm{l})$ brown inks. Scale bar $=5 \mathrm{~cm}$.

has generated additional diffraction spots. ${ }^{27}$ The violet laser beam illumination $(405 \mathrm{~nm})$ of the grating resulted in diffraction of the first order at $27^{\circ}$ and the second order at $73^{\circ}$, which agreed with the theoretical diffraction spots (eq 6). In addition, the secondary wave diffracted two more spots (Figure 3a). The laser beam $(532 \mathrm{~nm})$ produced diffraction of the first order at $39^{\circ}$, which was similar to the theoretical diffraction angle, whereas the secondary wave created two additional spots at $18^{\circ}$ and $63^{\circ}$ (Figure 3b). Additionally, the red laser beam $(635 \mathrm{~nm})$ resulted in the diffraction of light at $47^{\circ}$ with $2^{\circ}$ difference as compared to the theoretical diffraction value and the secondary wave diffraction was at $24^{\circ}$ (Figure 3c). The additional diffraction spots resulted from the high absorption of black ink and subsequent secondary standing wave. The black dye-based ink received one of the standing beam stronger $(532 \mathrm{~nm})$ than the other $(1064 \mathrm{~nm})$. The two wavelength could electromagnetically induce diffraction intensity based on the thickness and the medium of the grating. ${ }^{27,28}$ Although the black ink generated additional diffraction spots from the secondary standing wave, another experiment has been conducted on black ink with $\mathrm{Nd}$ :YAG laser beam $1064 \mathrm{~nm}$ and there was no sign of secondary grating diffraction (ESI Material, Supporting Information (SI) Figure S1).

The red and brown inks showed resemblance in the diffraction of the first order (Figure 3e,f, k,1). However, there was a slight change in the $(405 \mathrm{~nm})$ laser diffraction of the second order from the two ink gratings (Figure $3 \mathrm{~d}-\mathrm{j}$ ). The red ink has generated diffraction at $68^{\circ}$ and the brown ink diffraction was at $71^{\circ}$ due to 
Table 1. Diffraction from Ink Based Gratings Using Three Laser Beams (405 nm, $532 \mathrm{~nm}, 635 \mathrm{~nm}$ ) and Comparison with the Theoretical Values. C: Colour, L: Laser, Exp S: Experimental Secondary Laser, Exp P: Experimental Primary Laser, Ther.: Theoretical Values

\begin{tabular}{|c|c|c|c|c|c|c|c|}
\hline Ink-C. & L. $(\mathrm{nm})$ & Exp.S(1) & Exp.P(1) & Ther.(1) & Exp.S(2) & Exp.P(2) & Ther.(2) \\
\hline black & 405 & $13^{\circ}$ & $28^{\circ}$ & $28.8^{\circ}$ & $44^{\circ}$ & $73.2^{\circ}$ & $74.6^{\circ}$ \\
\hline black & 532 & $18^{\circ}$ & $39^{\circ}$ & $39.3^{\circ}$ & $63^{\circ}$ & & \\
\hline black & 635 & $22^{\circ}$ & $47^{\circ}$ & $49.1^{\circ}$ & & & \\
\hline red & 405 & & $28^{\circ}$ & $28.8^{\circ}$ & & $68^{\circ}$ & $74.6^{\circ}$ \\
\hline red & 532 & & $39^{\circ}$ & $39.3^{\circ}$ & & & \\
\hline red & 635 & & $49^{\circ}$ & $49.1^{\circ}$ & & & \\
\hline blue & 405 & & $29^{\circ}$ & $28.8^{\circ}$ & & $74^{\circ}$ & $74.6^{\circ}$ \\
\hline blue & 532 & $32^{\circ}$ & $39^{\circ}$ & $39.3^{\circ}$ & & & \\
\hline blue & 635 & & $50^{\circ}$ & $49.1^{\circ}$ & & & \\
\hline brown & 405 & & $28^{\circ}$ & $28.8^{\circ}$ & & $71^{\circ}$ & $74.6^{\circ}$ \\
\hline brown & 532 & & $39^{\circ}$ & $39.3^{\circ}$ & & & \\
\hline brown & 635 & & $49^{\circ}$ & $49.1^{\circ}$ & & & \\
\hline
\end{tabular}

the change reflective index of each color (eq 7 and 8). In addition, there were no signs of secondary grating diffraction because both inks gratings had low absorption profiles to produce a secondary grating (Figure $2 b-d$ ). Furthermore, the blue ink had slight differences on the diffraction points with an additional weak diffraction spot when illuminated with a $532 \mathrm{~nm}$ laser beam (Figure $3 \mathrm{~g}-\mathrm{i}$ ). In addition, most of the absorption range was within 560-650 nm, which should allow more diffraction intensity for a $635 \mathrm{~nm}$ laser beam (Figure 3i). However, the primary diffraction spot locations of the three laser beams on the blue ink grating closely matched with the calculated theoretical values (Table 1). As a result, the longer wavelength of laser light diffracted through a larger angle than the shorter wavelength laser light. In addition, the diffractions from the three lasers did not have identical positions even though the spacing of the gratings for the four inks were the same. This is because they have different refractive index of thick material. The experiment has been repeated multiple times using the four colors and the results showed that the material color has influenced the diffraction. The diffraction order $(\mathrm{m})$ can be theoretically estimated:

$$
\Lambda=\frac{\lambda m}{\sin (\theta)}
$$

Variation in monochromatic light wavelengths to illuminate ink gratings changed diffraction efficiency. The diffraction efficiency $\left(I_{\text {eff }}\right)$ of each ink grating was considered from the relation of the intensity of first order $I_{1}$ and the zero-order intensity $I_{0}$ and $\left(I_{\text {eff }}=I_{1} / I_{0}\right)$. The black ink had three equal monochromatic diffraction spots of the first order because of its absorption behavior of the broad wavelengths. However, the diffraction efficiencies of the three laser beams were not the same because some of the ink-based gratings had different light transmissions at zero-order (Figure 4a). The light diffraction efficiency of the red ink at $635 \mathrm{~nm}$ was the lowest because most light transmitted in the zero-order as the same wave color (Figure $4 \mathrm{~b})$. On the other hand, this was not the case for the blue ink. The light at $405 \mathrm{~nm}$ had high diffraction efficiency due to the two waves (primary and secondary) creating a higher sinusoidal absorption grating with small waves to produce a phase grating diffraction in the same position of the first order ${ }^{24,27}$ (Figure 4c). The brown ink light diffraction efficiency was the lowest because it had low light absorption (Figure 4d). The ink thickness had an important role on light diffraction based on absorption parameter $\left(Q^{\prime}\right)$ and refractive index effect can be calculated as
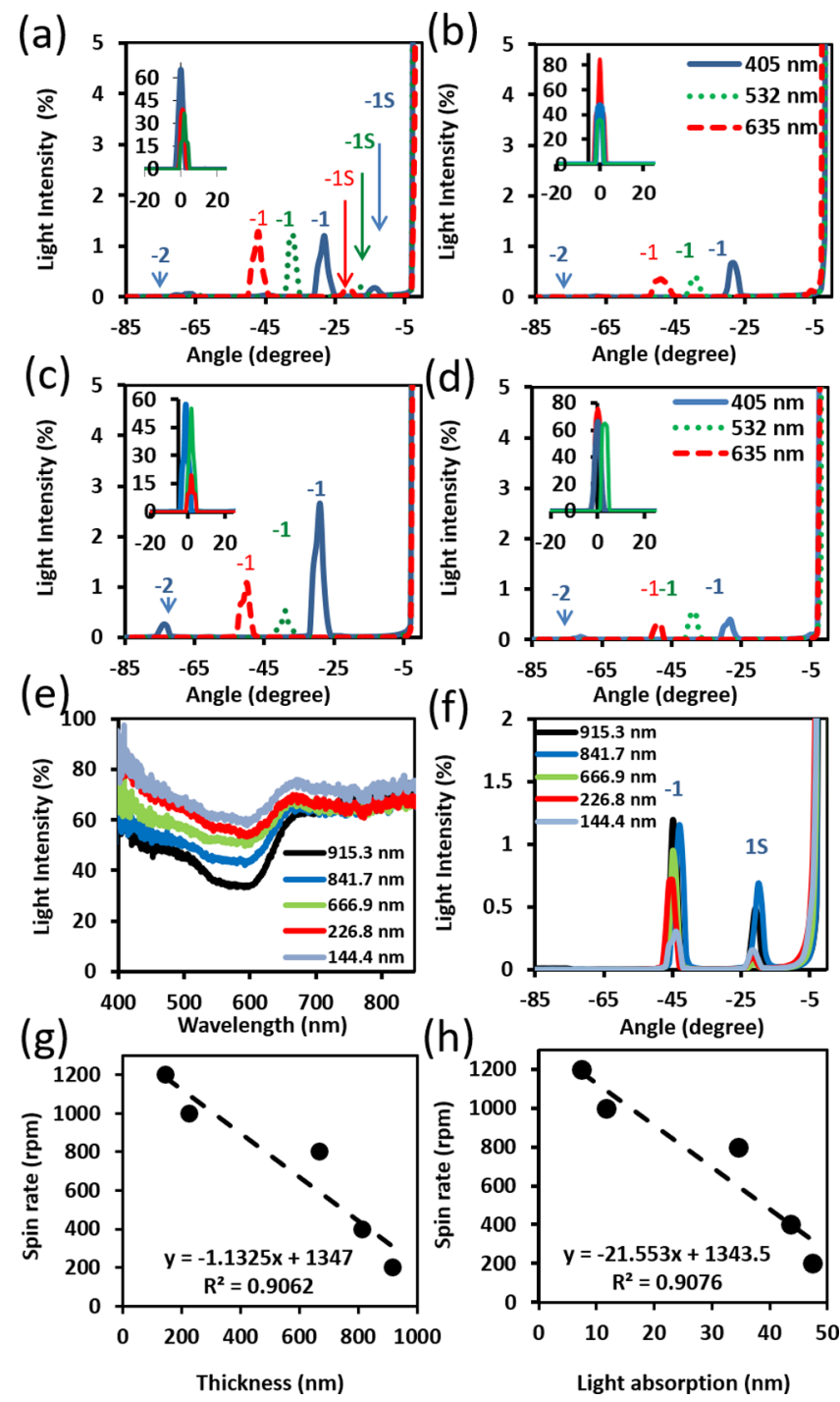

Figure 4. Diffraction intensity of the zero and first order from three monochromatic lights $(405,532$, and $635 \mathrm{~nm})$ on four patterned ink gratings. (a) Black, (b) red, (c) blue, and (d) brown ink gratings. Black ink gratings at different thicknesses in (e) transmission, (f) diffraction modes. Spin-coating effect (g) Thickness, (h) Light absorption.

$$
Q^{\prime}=2 \pi \lambda h / n_{\mathrm{o}} \Lambda^{2} \cos \theta
$$



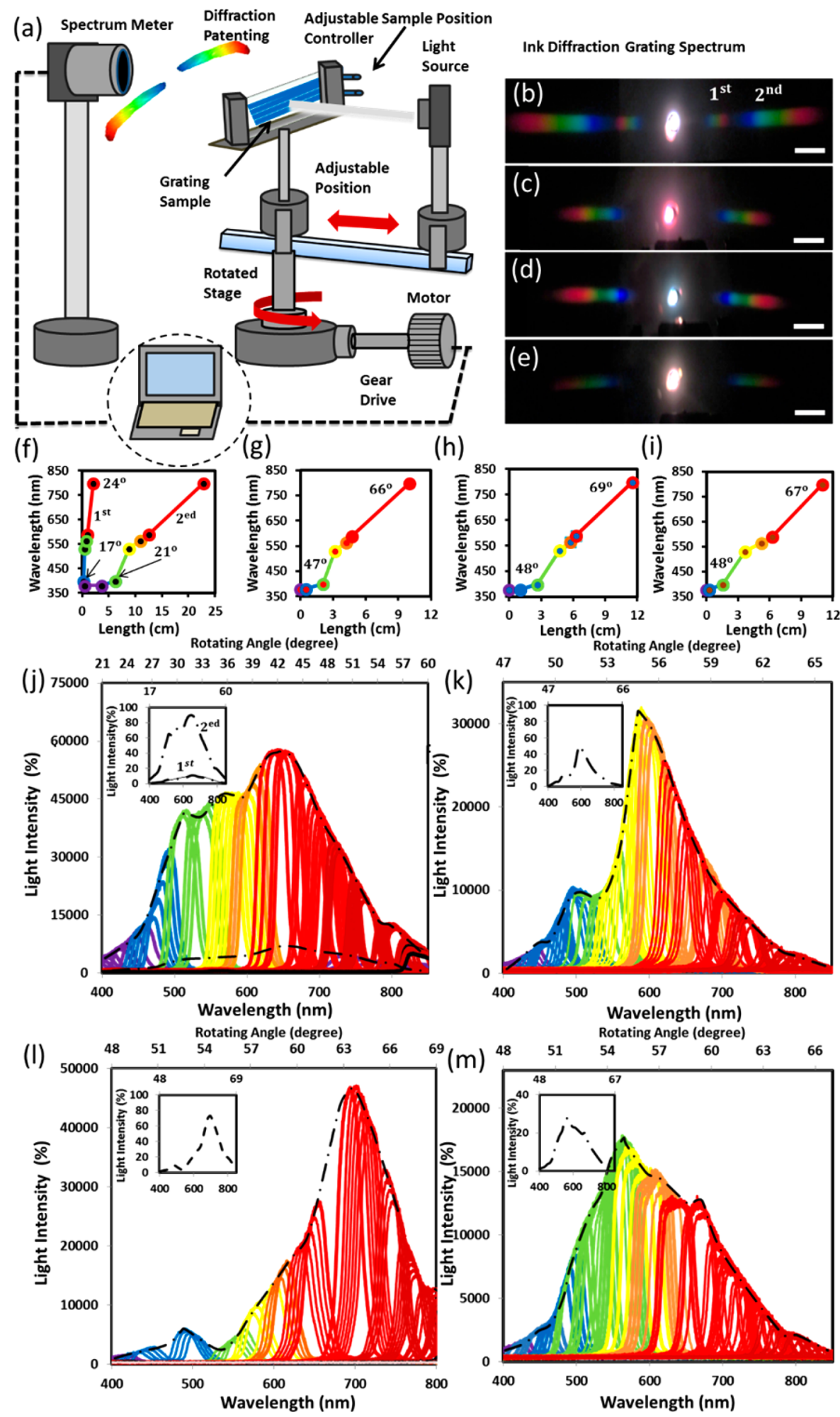

Ink Diffraction Grating Spectrum

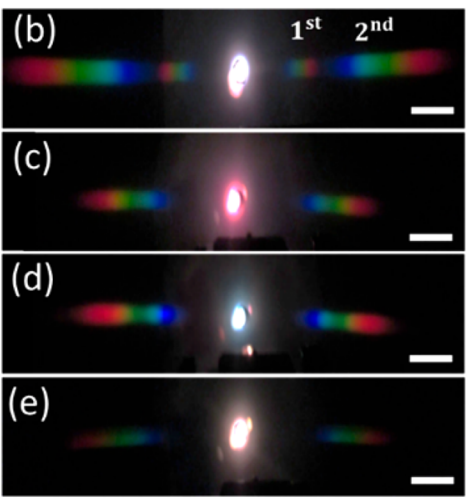

h)

(i)

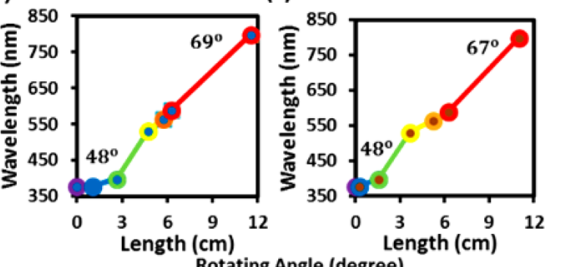

(k)

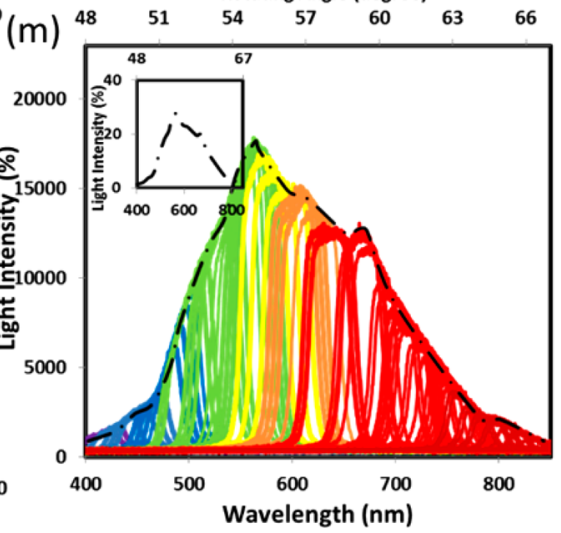

Figure 5. Angle-resolved measurements of the diffraction gratings fabricated via holographic DLIP. (a) The spectroscopy system contained the diffraction of a broadband light beam that passed through a $1 \mathrm{D}$ ink sample on a motorized stage to measure the rainbow pattern. The rainbow grating diffraction pattern of (b) black, (c) red, (d) blue, and (e) brown inks. Rainbow pattern length from the grating diffraction of (f) black, (g) red, (h) blue, and (i) brown light diffraction. Spectroscopy measurements of $(\mathrm{j})$ black, $(\mathrm{k})$ red, (l) blue, and ( $\mathrm{m}$ ) brown dye-based inks. Scale bare $=5 \mathrm{~cm}$.

$$
m=2 \pi n_{\mathrm{o}} \Lambda \sin \theta / \lambda
$$

where $Q^{\prime}$ is the thickness parameter, $h$ is the grating thickness, and $n_{\mathrm{o}}$ is the refractive index of the grating vector. ${ }^{24,29}$ Black ink was selected to test the effect of the thickness because it had equal transmission for three monochromatic laser lights. The laser light
$(635 \mathrm{~nm})$ was used to test the transmission profile of different thicknesses.

The black ink was spin-coated on $1 \mathrm{~mm}$ thick glasses at a speed of (200-1200 rpm). The transmission decreased with lower spin-coating speed and higher thickness (Figure 4e). Addition- 
ally, the diffraction intensity of first order increased with increasing thickness of the ink layer (Figure 4f). However, the secondary grating diffractions did not follow the first order trend and showed different responses in diffraction intensity. The first order diffraction is commonly used in optical applications because it has the highest efficiency, and in the present analysis, it can be the primary evaluation parameter. The thickness of the ink medium was being controlled by the spin-coating speed (Figure $4 \mathrm{~g}$ ). In addition, the ink layer thickness had a significant effect in light absorption (Figure 4h). As a result, increasing the ink thickness reduced the transmission, which enhanced the diffraction efficiency. The refractive index and absorption parameters at different thicknesses were calculated by (eq 7 and 8); and therefore, $915 \mathrm{~nm}$ thickness was chosen as the best medium thickness for the subsequent experiments. Using laser interference in Denisyuk reflection mode, the material thickness must be reasonable to allow the laser waves to pass through any substrate to produce the ablative reflection waves. If the material does not transmit the laser waves, the medium would burn it or not respond. In addition, if the absorption reaches zero or $100 \%$ transmission, the target material would not interact with laser wave. ${ }^{17,20}$ For these reasons, the ink thickness was sufficient to have reasonable absorption, in particular the black and blue inks.

A goniometer controlled spectroscopy setup was used for angular measurements and the distribution of the rainbow diffraction pattern from each ink grating was recorded (Figure 5a). The angle-resolved diffraction measurements of the four ink gratings were measured at a maximum broadband light intensity to quantify the highest value of diffracted wavelength distribution. The maximization enabled to have the same bandwidth of grating diffraction within the range of 400-850 $\mathrm{nm}$. The rainbow diffraction of each ink grating had different arcs and number of orders. The length measurements were based on placing a screen at $30 \mathrm{~cm}$ away from diffraction samples. The black ink grating had the widest dispersion pattern as compared to the other inks, and it produced two diffraction orders with a total angular diffraction of $43^{\circ}$ from one side of the rotation stage $\left(0^{\circ}\right.$ to $\left.+90^{\circ}\right)$. The total length of the two orders was $22.83 \mathrm{~cm}$ (Figure $5 b-f$ ). The red and brown ink gratings had the same total angular diffraction having a width of $19^{\circ}$ to $(10 \mathrm{~cm}$ long) (Figure $5 \mathrm{c}-\mathrm{g}$ and $5 \mathrm{e}-\mathrm{i}$ ). However, the blue ink formed a different shape of the rainbow to create a thinner width at the green region with a total diffraction of $23^{\circ}$ and $12.7 \mathrm{~cm}$ long (Figure $5 \mathrm{~d}-\mathrm{h}$ ). The black ink generated two rainbow patterns: the first order was $17^{\circ}-22^{\circ}$ and the second order was at $21^{\circ}-60^{\circ}$ from the two laser waves (Figure 5f). The black ink had the highest diffraction intensity of all light waves due to high light absorption (Figure $5 \mathrm{j}$ ), but $11 \%$ of light waves was diffracted at the first order (ESI Material (SI) Figure S2). However, the red ink diffracted less light from the rainbow pattern because it had low light absorption (Figure $5 \mathrm{~g}$ ) and diffraction concentrated at the peaks at 490 to $625 \mathrm{~nm}$ based on its absorption zone (Figure $5 \mathrm{k}$ ). The blue ink grating had the highest diffraction value of $73 \%$ because of the light absorption between the two peaks (Figure 51). Nevertheless, the lowest intensity of wavelength distribution was with the brown ink, reaching a maximum of $28 \%$ diffraction (Figure 5i). This is because brown medium lost most of light to transmission at the zero-order that led to a low percentage of overall light diffraction (Figure 5m) (ESI Material, SI Figure S3). Consequently, the four ink gratings showed a different response on the rainbow diffraction pattern. The blue and red grating inks indicated that both of them were able to create single wavelength low-cost photonic structures with wavelength dependence.
However, the black and brown inks were not able to generate specific wavelength filtering because both inks diffracted light in all wavelengths.

Applications of the Ink-Based Gratings. The dye-based inks on glass substrates could be used as low-cost wavelength filters. Their thickness of the ink layer could be controlled to adjust the percentage of light transmission and to create different photonic effects (Figure 6a1-a4). The numbers of laser pulses
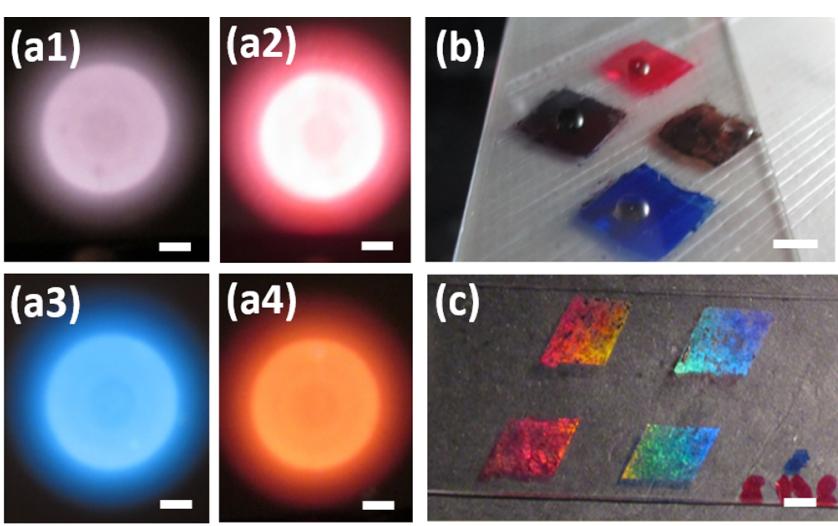

$83^{\circ}$
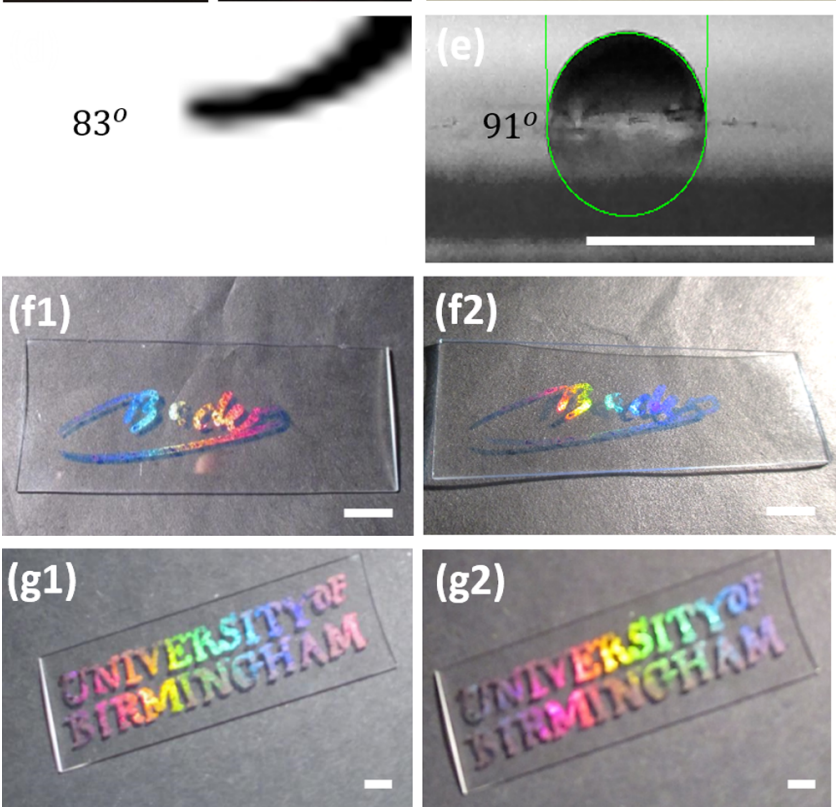

Figure 6. Hydrophobic properties and applications of dye-based ink gratings. (a1) black, (a2) red, (a3) blue, and (a4) brown ink wavelength filters. (b) Hydrophobicity measurements on the dye-based inks. (c) The nanopatterned gratings in array form. $(\mathrm{d}, \mathrm{e})$ hydrophobicity contact angle change. ( $\mathrm{f} 1, \mathrm{f} 2)$ Blue ink based $1 \mathrm{D}$ grating personal security signature. (g1,g2) Black ink based surface grating University of Birmingham logo. Scale bar $=5 \mathrm{~mm}$.

were varied on the dye-based inks to filter transmission but this process ablated most of the ink medium to create $\sim 90 \%$. The laser energy and the thickness of the recording medium should be controlled to finely tune the optical properties of the wavelength filter transmission. In addition, the hydrophobicity measurements were carried out on the ink gratings with deionized water (Figure $6 b$ ). The nanopatterned gratings were created on the four ink types (Figure 6c) to show the visible effect of nanostructures and to create 1D structures (800 nm periodicity) for the hydrophobicity measurements. The hydrophobicity has increased after the nanostructure formation on the 
dye-based inks, but possibility of less than $10 \%$ of difference from dye ink gratings color and instrument accuracy precision. We also demonstrate this capability by creating $1 \mathrm{D}$ nanostructures in blue ink (Figure 6d,e). In addition, there are many other optical applications that can be achieved with dye-based ink gratings. Multiple laser exposures were conducted to ablate the ink substrate in a $\mathrm{x}-\mathrm{y}$ linear movement stage at $15^{\circ}$ to produce a $1 \mathrm{D}$ diffraction grating in the form of a signature. A $1 \mathrm{D}$ grating based signature with blue ink can be used to create a high security feature (Figure $6 \mathrm{fl} 1, \mathfrak{f} 2$ ). According the manufacturer, the inks have long-term durability. In these experiments, there was no change in sample quality after a year. The sample shown in Figure $6 \mathrm{f} 1$ is one year older than the sample in Figure $6 \mathrm{f} 2$, and they both have comparable optical performances. Moreover, a 1D diffraction grating based logo of "University of Birmingham" was created by ablating black ink as examples of sophisticated design of trademark and brands for diverse commercial applications (Figure $6 \mathrm{~g} 1, \mathrm{~g} 2$ ). Their applications in optical devices include holographic displays, data storage, filters, light trapping, security systems, and sensors. ${ }^{21,30,31}$

\section{CONCLUSIONS}

We report the production of optical nanogratings based on economical dye-based inks. The diffraction from these gratings depended on the ink's refractive index and incident wavelength. The black ink based nanostructures displayed additional diffraction spots from its secondary grating, whereas the phase grating in the blue nanostructure enhanced light diffraction of the same wavelength. Moreover, with increasing ink thickness, the light diffraction is enhanced and the effective transmission is decreased. In addition, the ink based gratings can be used as a wavelength-dependent filter and their thickness can be controlled to adjust the percentage of transmission. In particular, the blue and red ink nanostructures have the potential to be used as low cost wavelength-dependent photonic structures, which have application in displays, fiber optics, and biosensors. Furthermore, hydrophobicity measurements were performed on the four ink gratings to show the increase in the contact angle due to the topology of the nanostructures. To demonstrate the utility, the blue ink based grating was demonstrated as an example of an advanced personal security signature, and a black ink grating was patterned to display "University of Birmingham" as a distinctive logo. The DLIP patterning method described herein has wide potential applications in commercial diffraction optics.

\section{ASSOCIATED CONTENT}

\section{S Supporting Information}

The Supporting Information is available free of charge on the ACS Publications website at DOI: 10.1021/acsami.7b15713.

Additional information as noted in the text (PDF)

\section{AUTHOR INFORMATION}

\section{Corresponding Author}

*Phone: +441214158623; e-mail: h.butt@bham.ac.uk.

\section{ORCID $\odot$}

Ali K. Yetisen: 0000-0003-0896-267X

\section{Notes}

The authors declare no competing financial interest.

\section{ACKNOWLEDGMENTS}

We acknowledge Leverhulme Trust and Wellcome Trust for the research funding.

\section{REFERENCES}

(1) Zgirski, M.; et al. Ion beam shaping and downsizing of nanostructures. Nanotechnology 2008, 19 (5), 055301.

(2) Sabouri, A.; et al. Effects of current on early stages of focused ion beam nano-machining. Mater. Res. Express 2015, 2 (5), 055005.

(3) Kong, X.-T.; et al. Graphene-based ultrathin flat lenses. ACS Photonics 2015, 2 (2), 200-207.

(4) Alqurashi, T.; et al. Femtosecond laser directed fabrication of optical diffusers. RSC Adv. 2017, 7 (29), 18019-18023.

(5) Alqurashi, T.; et al. Nanosecond pulsed laser texturing of optical diffusers. AIP Adv. 2017, 7 (2), 025313.

(6) Zhao, Q.; et al. Printable nanophotonic devices via holographic laser ablation. ACS Nano 2015, 9 (9), 9062-9069.

(7) Zhu, X.; et al. Resonant laser printing of structural colors on highindex dielectric metasurfaces. Science Advances 2017, 3 (5), e1602487.

(8) Højlund-Nielsen, E.; et al. Plasmonic Colors: toward mass production of metasurfaces. Advanced Materials Technologies 2016, 1 (7), 1600054-n/a.

(9) Xiong, K.; et al. Plasmonic metasurfaces with conjugated polymers for flexible electronic paper in color. Adv. Mater. 2016, 28 (45), 99569960.

(10) Huang, Y.; et al. Printable functional chips based on nanoparticle assembly. Small 2017, 13 (4), 1503339-n/a.

(11) Medintz, I. L.; et al. Quantum dot bioconjugates for imaging, labelling and sensing. Nat. Mater. 2005, 4 (6), 435.

(12) Zhang, X.-Y.; et al. Self-assembly of large-scale and ultrathin silver nanoplate films with tunable plasmon resonance properties. ACS Nano 2011, 5 (11), 9082-9092.

(13) Zheng, Z. G., et al., Light-patterned crystallographic direction of a self-organized $3 D$ soft photonic crystal. Adv. Mater., 2017.170316510.1002/adma.201703165

(14) Sun, P.-z.; et al. Light-reconfigured waveband-selective diffraction device enabled by micro-patterning of a photoresponsive self-organized helical superstructure. J. Mater. Chem. C 2016, 4 (39), 9325-9330.

(15) Zhu, X.; et al. Plasmonic colour laser printing. Nat. Nanotechnol. 2016, 11 (4), 325-329.

(16) Carstensen, M. S.; et al. Holographic Resonant Laser Printing of flat optics using template plasmonic metasurfaces. arXiv preprint arXiv 1708, 05571, 2017.

(17) Deying Xia.; et al. Nanostructures and functional materials fabricated by interferometric lithography. Adv. Mater. 2011, 23 (2), 147-179.

(18) Karnakis, D. M. High power single-shot laser ablation of silicon with nanosecond 355nm. Appl. Surf. Sci. 2006, 252 (22), 7823-7825.

(19) Zhao, Q.; et al. Printable ink holograms. Appl. Phys. Lett. 2015, 107 (4), 041115.

(20) AlQattan, B.; et al. Holographic direct pulsed laser writing of twodimensional nanostructures. RSC Adv. 2016, 6 (112), 111269.

(21) Vasconcellos, F.d.C.; et al. Printable surface holograms via laser ablation. ACS Photonics 2014, 1 (6), 489-495.

(22) Ahmed, R.; et al. Printable ink lenses, diffusers, and 2D gratings. Nanoscale 2017, 9 (1), 266-276.

(23) Magnusson, R.; Gaylord, T. Solutions of the thin phase grating diffraction equation. Opt. Commun. 1978, 25 (2), 129-132.

(24) Magnusson, R.; Gaylord, T. Diffraction efficiencies of thin absorption and transmittance gratings. Opt. Commun. 1979, 28 (1), 13.

(25) Magnusson, R.; Gaylord, T. Analysis of multiwave diffraction of thick gratings. J. Opt. Soc. Am. 1977, 67 (9), 1165-1170.

(26) Hecht, E.; Zajac, A. Optics Addison-Wesley. Reading, Mass 1974, 19872, 350-351.

(27) Naseri, T.; Sadighi-Bonabi, R. Efficient electromagnetically induced phase grating via quantum interference in a four-level N-type atomic system. J. Opt. Soc. Am. B 2014, 31 (10), 2430-2437. 
(28) Gao, H.; et al. Analysis on diffraction properties of the transmission phase grating. Optik 2007, 118 (9), 452-456.

(29) Magnusson, R; Gaylord, T. Diffraction regimes of transmission gratings. J. Opt. Soc. Am. 1978, 68 (6), 809-814.

(30) Blanche, P.-A.; et al. Holographic three-dimensional telepresence using large-area photorefractive polymer. Nature 2010, 468 (7320), 8083.

(31) Xia, D.; et al. Nanostructures and functional materials fabricated by interferometric lithography. Adv. Mater. 2011, 23 (2), 147-179. 